\title{
Interaction between Primary Care Physicians and Specialists for Diagnosis and Management of Hypersensitivity Pneumonitis
}

\author{
Keiko Morii $^{1,2,3}$, Kozo Yoshimori ${ }^{1}$, Miyako Sudo ${ }^{1}$, Hideo Ogata ${ }^{1}$, Masao Okumura ${ }^{1}$, Akihiko Gemma ${ }^{3}$, \\ Shoji Kudoh ${ }^{1}$, Kozui Kida ${ }^{2,3}$ \\ ${ }^{1}$ Department of Pulmonary Diseases, Fukujuji Hospital, Kiyose City, Tokyo, Japan; ${ }^{2}$ The Respiratory Care Clinic, Nippon Medical \\ School, Tokyo, Japan; ${ }^{3}$ Department of Internal Medicine, Division of Pulmonary Medicine, Infectious Diseases and Oncology, Nip- \\ pon Medical School, Tokyo, Japan. \\ Email:kkida@nms.ac.jp
}

Received June $13^{\text {th }}, 2011$; revised July $28^{\text {th }}, 2011$; accepted August $10^{\text {th }}, 2011$.

\begin{abstract}
Objective: Hypersensitivity pneumonitis (HP) may be a complex syndrome rather than a single, uniform disease entity. The problems associated with HP treatment include a lack of awareness of primary care procedures and scarcity of recent information regarding HP. The main objective of this study was to investigate the problems in the interaction between primary care physicians and chest specialists. Data source: All available clinical records of cases at the Fukujuji Chest Hospital, Tokyo, between 1994 and 2005, supervised by specialists of a university hospital. Study selection: All cases suspected of HP during the period. Results: Nine cases were excluded because of insufficient records or because they did not satisfy the clinical criteria. Twenty-eight enrolled patients (14 men and 14 women; mean age, 53.0 years) were initially treated for respiratory infections by primary care physicians. The final HP types were summer-type $(n=18)$, bird fancier's lung $(n=2)$, ventilation-related $(n=3)$, or undetectable antigen $(n=5)$. On the basis of the interval between the onset of initial symptoms and the time of referral to our hospital, the cases can be categorized into 3 groups, which may represent acute, subacute, and chronic HP. Conclusion: All patients initially received treatment on the basis of a different diagnosis at primary evaluation. We concluded that interaction between primary care physicians and chest specialists is essential for solving problems associated with the early diagnosis and adequate treatment of HP.
\end{abstract}

Keywords: Biopsy, Connection, Computed Tomography, Criteria, Pneumonitis

\section{Introduction}

Hypersensitivity pneumonitis (HP) is a complex syndrome of varying intensity, clinical presentation, and natural history rather than a single, uniform disease entity [1-3]. On the basis of a nationwide epidemiological study, the Japanese Research Committee on Diffuse Pulmonary Disease for Hypersensitivity Pneumonitis has proposed diagnostic criteria for HP [4-6]. The survey, which was sponsored by the Japanese Ministry of Health, Labor and Welfare, revealed that summer-type HP (S-HP) is the most common type of HP in Japan. Of the 835 surveyed patients, 621 (74.4\%) were diagnosed with S-HP [4]. According to its clinical presentation, HP can be categorized as acute, subacute, or chronic illness $[1,7,8]$. Although there may be a considerable overlap among these
3 types of HP, this classification is a useful approach for categorizing the variations in clinical presentations that are seen in this disease $[8,9]$. With regard to research on HP, 2 practical issues need to be pointed out: one is a lack of sufficient information on the clinical criteria for chronic or subacute types of S-HP [8,9] and another is the inadequate collaboration between primary care physicians and core community hospital staff in terms of clinical service for HP. HP is rarely encountered during primary care; however, when it is suspected, the patient might be referred for secondary care or to a hospital for chest diseases. Our hospital, which specializes in chest diseases, is located outside the central Tokyo area, and receives patients as a core hospital and as referrals from local clinics as well as from clinics in the central area of Tokyo. 
In this study, we studied these issues in HP diagnosis in patients who were diagnosed with HP over a 10-year period. Furthermore, we investigated the role of interactive communication between primary care physicians and specialists and revisited the diagnostic criteria for HP in Japan.

\section{Methods and Patients}

\subsection{Case Selection and Examinations}

All patients who were suspected of having HP on the basis of clinical diagnosis and were hospitalized at the Fukujuji Chest Hospital, Tokyo, between January 1994 and November 2005 were included in this study. The diagnosis of HP was made on the basis of exposure history and the results of clinical assessments, including blood analysis and determination of KL-6 levels, which served as a specific biomarker for interstitial lung disease [10]. In addition, high-resolution computed tomography (HRCT) images of the chest were analyzed. The effect of removing the patient from the suspected etiologic exposure was also considered. Other tests such as bronchoalveolar lavage and transbronchial lung biopsy were used to rule out other potential diagnoses and to lend further support to the diagnosis of HP. This retrospective study was performed by taking into account all available clinical records described above.

\subsection{Diagnostic Criteria}

The diagnosis of HP in this study was essentially based on the diagnostic criteria of the Japanese Research Committee on Diffuse Pulmonary Disease for Hypersensitivity Pneumonitis, [6] which are similar to criteria described in previous studies [1,7,11-14]. Acute, subacute, or chronic HP [1,7-9] and the possible causes of HP [1,6, 7,11-14] were determined by performing previously reported procedures $[8,9]$. Summer-type, bird-fancier's lung, or ventilator-related types of HP were also determined using these protocols.

\subsubsection{Compatible Clinical and Environmental Information}

Patients who showed respiratory and/or constitutional symptoms and signs such as crackles on chest auscultation, cough, breathlessness, febrile episodes, wheezing, and fatigue were enrolled as possible cases of HP. Particular attention was paid to the assessment of each clinical course in terms of acute, subacute, or chronic illness described above.

\subsubsection{Laboratory Testing and Radiographic Findings}

Reticular, nodular, or ground-glass opacity on chest radiographs or HRCT images comparable with the opaci- ties reported in a previous study on HP were assessed [14]. Appropriate laboratory tests, including KL-6 detection when available, were performed on initial examination [10]. The presence of specific serum IgG antibodies against the identified antigen (serum precipitins) was reviewed. Exposure to known offending antigen (s) was determined on the basis of a history of appropriate exposure or aerobiologic or microbiologic investigations of the environment to confirm the presence of an inciting antigen. On the basis of the recommendations for the diagnosis of S-HP in Japan, serum precipitins for the following 4 organisms were measured in patients suspected of having HP: Trichosporon (T) cutaneum, T. asahii, T. domesticum, and Cryptococcus [4,5,9].

\subsubsection{Bronchoalveolar Fluid and Lung Pathology}

Lymphocytosis in bronchoalveolar lavage fluid (BALF) and the CD4/CD8 ratio [13] were examined. Histopathology showed compatible changes, which included pathological findings of poorly formed, non-caseating granulomas or mononuclear cell infiltration [13].

\subsubsection{Challenge Tests}

The findings of these tests are especially suggestive if they are present, appearing, or worsening several hours after antigen exposure [13]. A positive inhalation challenge test based on re-exposure to the environment was conducted; in this test, the subject was required to stay at his/her home/workplace for more than $12 \mathrm{~h}$. In this study, subjects suspected of having S-HP underwent returnhome testing [6].

\subsection{Statistics}

Data were analyzed using the Statistical Package for the Social Sciences (SPSS) Version 11.1 for Windows (SPSS Inc., Chicago, Illinois, U.S.A.). We used Student's $t$ test or analysis of variance for continuous variables and the chi-square test or Fisher's exact test for categorical variables. Data are expressed as mean $\pm 1 \mathrm{SD}$; all reported $\mathrm{P}$-values are 2-sided, and $\mathrm{P}$ values less than 0.05 were considered significant.

\section{Results}

Over 10 consecutive years, a total of 37 patients received a clinical diagnosis of HP. Among these, 3 patients were excluded from our study because of insufficient data, and 6 patients whose data did not satisfy the clinical criteria were also excluded. Thus, a total of 28 cases were assessed in the study.

\subsection{Diagnosis and Treatment by Primary Care Physicians before Admission}

All 28 patients were treated by primary care physicians 
before admission to our hospital. The initial diagnoses made by the primary care physicians were as follows: acute upper respiratory infection $(\mathrm{n}=4)$, bronchitis $(\mathrm{n}=$ 11), pneumonia caused by Mycoplasma infection $(\mathrm{n}=1)$, bacterial pneumonia $(\mathrm{n}=3)$, interstitial pneumonia $(\mathrm{n}=$ 5), HP $(\mathrm{n}=1)$, bronchial asthma $(\mathrm{n}=1)$, and undetermined diagnosis $(n=2)$. The mean interval between the initial clinical symptoms and referral to our hospital was $10.5 \pm 8.3 \mathrm{wk}$. The distribution of this interval is shown in Figure 1, which roughly shows at least 3 groups; short, intermediate, and long interval. In this study, we assumed that the short, intermediate, and long interval groups corresponded with acute $(n=19)$, subacute $(n=4)$, and chronic $(\mathrm{n}=2)$ disease, respectively. Three cases could not be classified into any of the groups. The medications prescribed by the primary care physicians were as follows: none $(\mathrm{n}=2)$, antibiotics $(\mathrm{n}=21)$, bronchodilator $(\mathrm{n}=3)$, cough medicine $(\mathrm{n}=6)$, systemic steroid $(\mathrm{n}=2)$, and unknown $(\mathrm{n}=2)$ (redundant counts were allowed).

\subsection{Clinical History, Initial Symptoms, and Physical Findings after Admission}

The subjects included 14 men and 14 women with a mean age of 53.0 years (range: 22 - 74 years). The subjective symptoms at the initial visit to the institute were cough (17.9\%), fever (46.4\%), and dyspnea either at rest or on exertion (21.4\%). Information on the place of work just before the onset of symptoms was available in 22 patients, including householders $(n=9)$, company em- ployees $(n=7)$, and office workers $(n=6)$, but 6 patients did not show an obvious or known exposure to specific agents. Eleven patients smoked or had a history of smoking. Three patients had underlying lung diseases, including bronchial asthma $(\mathrm{n}=2)$ and chronic obstructive pulmonary disease (COPD; $\mathrm{n}=1)$. In 2 cases, one each in the short- and the long-interval groups, an initial diagnosis of interstitial pulmonary fibrosis (IPF) was made on the basis of the HRCT images (see below). All chest radiographs were evaluated by both radiologists and chest specialist. Each radiograph was carefully re-assessed by 3 authors.

\subsection{Data after Admission}

The percentages of eosinophils and lymphocytes in the peripheral blood were $4.6 \% \pm 4.7 \%$ and $19.5 \% \pm 9.9 \%$, respectively. Cell populations in the peripheral blood showed significant differences among the various types of HP, e.g., the number of white blood cells in the ventilator-related type was greater than those in the bird fancier's lung type $(\mathrm{p}=0.033)$ and undetectable antigen type $(p=0.003)$. However, such differences were not observed in the number of neutrophils or lymphocytes. Furthermore, the proportion of neutrophils was greater in the cases of acute HP (acute vs. chronic, $p=0.028$ ), while the proportion of lymphocytes was smaller in the same disease group (acute vs. subacute, $\mathrm{p}=0.010$; acute vs. chronic, $\mathrm{p}=0.003$ ).

Serum-specific antibodies (precipitins) were detected

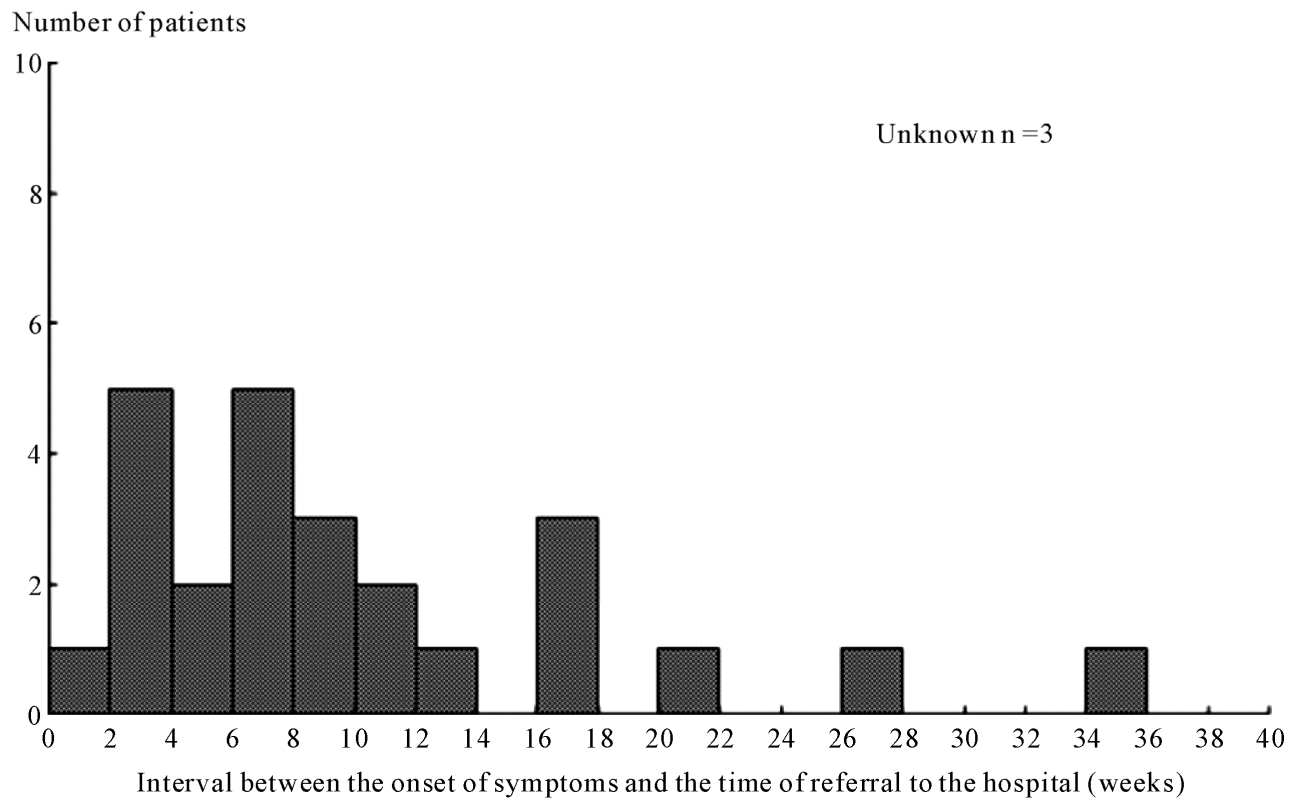

Figure 1. Distribution of the mean interval between onset of initial symptoms and referral to our hospital. Acute, subacute, and chronic illness categories may be reflective of the interval between onset of symptoms and time of referral to our hospital, namely short (0 wk - 14 wk), intermediate (15 wk - 24 wk), and long interval ( $>25$ wk). 
in 13 patients; this included antibodies for antigens of $T$. cutaneum $(\mathrm{n}=9 ; 32.1 \%), T$. asahii $(\mathrm{n}=6 ; 21.4 \%), T$. domesticum ( $\mathrm{n}=4 ; 14.3 \%)$, and Cryptococcus $(\mathrm{n}=8$; $28.6 \%$ ).

Data on KL-6 levels were available for 17 patients. The mean KL-6 concentration was $2386 \mathrm{U} / \mathrm{ml}$ (range: 215 - $8580 \mathrm{U} / \mathrm{ml}$; normal range: less than $499 \mathrm{U} / \mathrm{ml}$ ). As shown in Figure 2, cases with KL-6 levels between 500 and $5000 \mathrm{U} / \mathrm{ml}$ were compatible with various types of HP. Nonetheless, these also included cases with undetectable levels of serum precipitin (s). Serum concentration of KL-6 was determined in 19 cases; the sensitivity was $82.4 \%$, however the specificity was unable to calculate, because of insufficient samples.

\subsection{HRCT Findings}

A summary of the HRCT findings is shown in Table $\mathbf{1 .}$ In 1 case, which was diagnosed as idiopathic pulmonary fibrosis by the primary care physician, a honeycomb appearance was observed on the HRCT image; a similar finding was obtained in another case. However, none of the other cases showed such HRCT findings.

\subsection{BALF, Lung Pathology, and Outcome of Challenge Tests}

Table 2 shows a summary of the pathological findings for cellular components in the lung samples and BALF samples. The percentages of lymphocytes $(p=0.008)$ and eosinophils $(p=0.045)$ in BALF significantly increased with granuloma formation or Masson's body formation with eosinophil components $(p=0.039)$ in the lung samples. However, there was no correlation between the percentages of eosinophils in blood and BALF. No significant differences were found among the CD4/ CD8 ratios in BALF in S-HP $(0.43 \pm 0.29)$, ventilatorrelated $\mathrm{HP}(0.43 \pm 0.21)$, and undetectable antigen group $(0.21 \pm 0.09)$.

Figure 3 shows a comparison of the types of HP, defined according to the clinical criteria of the Japanese Research Committee on Diffuse Pulmonary Disease for Hypersensitivity Pneumonitis (1990), found in the present study and in a nationwide epidemiological study [4, 5]. The prevalence of S-HP in the present study was lower than that reported previously.

\subsection{Challenge Tests}

Fourteen patients showed re-activation on the returnhome test. A summary of the data is shown in Table 3.

\subsection{Treatments during Hospitalization, Outcomes, and Final Diagnosis}

Fifteen patients received systemic steroids; among these,

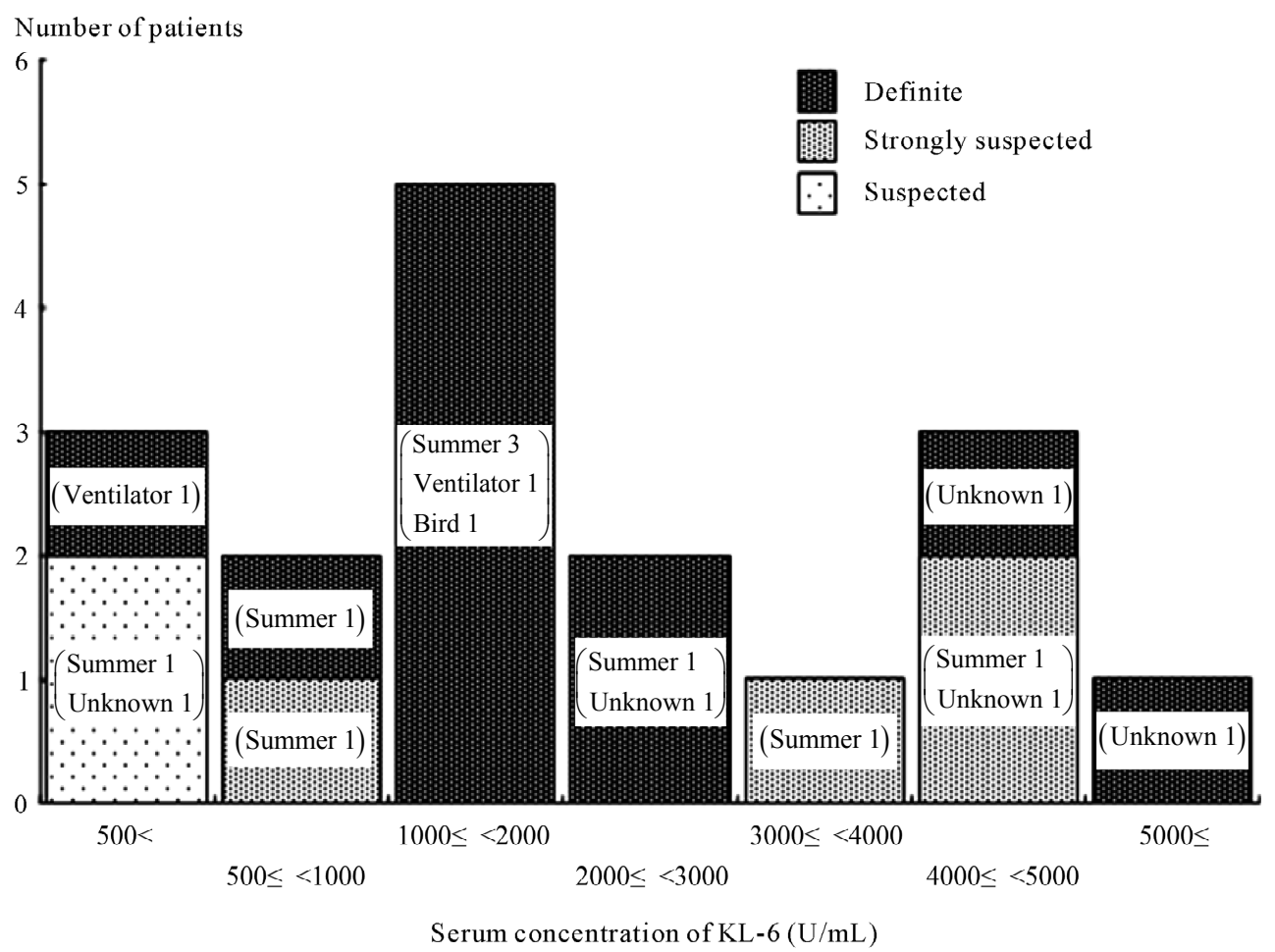

Figure 2. Distribution of serum concentration of KL-6. There were no discernable correlations between the serum KL-6 levels and the cause of HP in the 17 patients who underwent a KL-6 test. 


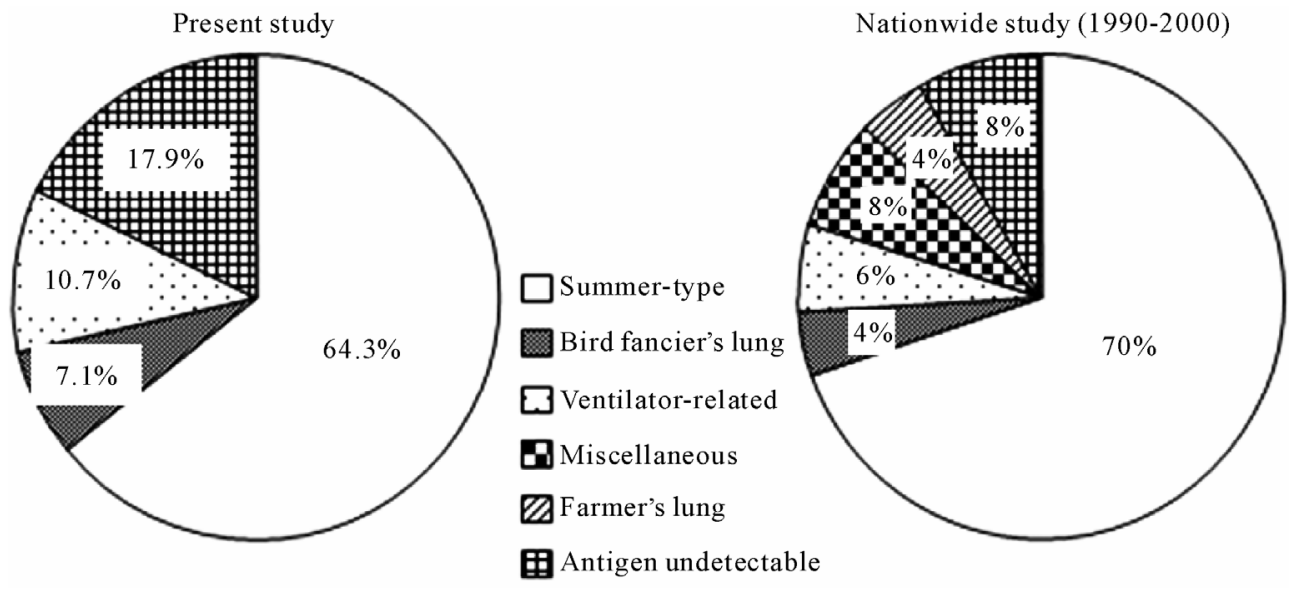

Figure 3. Comparison between the data obtained in the present study and those obtained in a nationwide epidemiological study. The prevalence of summer-type hypersensitivity pneumonitis was lower in our study cohort. Note the increase in patients with undetectable antigen and patients who were excluded from a diagnosis of hypersensitivity pneumonitis in our study (see text for details).

Table 1. Findings obtained on high-resolution computed tomography images.

1. Centriacinar nodular shadows: positive ratio $=17 / 28(60.7 \%)$

Positive group: Definite $\mathrm{HP} \dagger(\mathrm{n}=11)$, strongly suspected HP $(\mathrm{n}=4)$, suspected HP $(\mathrm{n}=2)$

Negative group: Definite HP $(n=8)$, strongly suspected HP $(n=2)$, suspected HP $(n=1)$

2. Ground-glass opacity (GGO): positive ratio $=26 / 28(92.9 \%)$

Positive group: Definite HP $(n=18)$, strongly suspected HP $(n=6)$, suspected HP $(n=2)$

Negative group: Definite HP $(n=1)$, strongly suspected HP $(n=0)$, suspected HP $(n=1)$

3. Thickness of acinar septum: positive ratio $=24 / 28(70.6 \%)$

Positive group: Definite HP $(n=15)$, strongly suspected HP $(n=6)$, suspected HP $(n=3)$

Negative group: Definite HP $(n=4)$, strongly suspected HP $(n=0)$, suspected HP $(n=0)$

Table 2. Pathological findings of lung samples and bronchoalveolar lavage fluid and findings obtained on high-resolution computed tomography in all cases $(n=28)$.

\begin{tabular}{crc}
\hline \multicolumn{3}{c}{ Lung pathology } \\
\hline Granuloma & 14 & $(50.0 \%)$ \\
Alveolitis & 22 & $(78.6 \%)$ \\
Masson's body & 7 & $(25.0 \%)$ \\
\hline \multicolumn{3}{c}{ Blood } \\
\hline Eosinophils (\%) & $4.6 \pm 4.7$ \\
Lymphocytes (\%) & $19.5 \pm 9.9$ \\
\hline \multicolumn{3}{c}{ BALF† } \\
\hline Lymphocytes (\%) & $65.3 \pm 26.3$ \\
Eosinophils (\%) & $3.4 \pm 3.9$ \\
CD4/CD8 & $0.42 \pm 0.30$ \\
\hline
\end{tabular}

$\dagger \mathrm{BALF}=$ bronchoalveolar lavage fluid.
8 underwent pulse administration. HP in 17 patients was resolved by improving environmental factors, i.e., moving to new housing $(\mathrm{n}=4)$, cleaning the house $(\mathrm{n}=2)$, renovating the house $(\mathrm{n}=4)$, and avoiding the antigens $(\mathrm{n}=7)$. Unknown factors were involved in 8 cases. According to the overall data stated above, the final diagnoses were as follows: summer-type HP $(n=18)$ : definite $(\mathrm{n}=12)$, strongly suspected $(\mathrm{n}=5)$, and suspected $(\mathrm{n}=1)$; bird fancier's lung $(\mathrm{n}=2)$ : definite $(\mathrm{n}=2)$; ventilator-related HP $(\mathrm{n}=3)$ : definite $(\mathrm{n}=2)$ and suspected $(\mathrm{n}=1)$; undetectable antigen $(\mathrm{n}=5)$ : definite $(\mathrm{n}=3)$, strongly suspected $(\mathrm{n}=1)$, and suspected $(\mathrm{n}=1)$.

\section{Discussion}

In the present study, we found that all patients were initially treated on the basis of a different diagnosis in the primary practice. Further, we found that the prevalence of summer-type HP in the present study was $64.3 \%$, 
Table 3. Pathological findings from lung samples and bronchoalveolar lavage fluid and hematological characteristics.

\begin{tabular}{|c|c|c|}
\hline \multicolumn{3}{|c|}{$\mathrm{n}=23$ (summer-type: 16$)$} \\
\hline \multicolumn{2}{|c|}{ Positive $(61 \%)$} & 14 (summer-type: 9) \\
\hline \multicolumn{2}{|c|}{ Negative $(39 \%)$} & 9 (summer-type: 7) \\
\hline \multicolumn{3}{|c|}{ Characteristic findings based on positive cases $(n=14)$} \\
\hline \multirow[t]{3}{*}{ Clinical Symptoms } & Cough & $5(35.7 \%)$ \\
\hline & Fever & $13(92.9 \%)$ \\
\hline & Dyspnea & $6(42.9 \%)$ \\
\hline \multirow[t]{3}{*}{ Blood chemistry } & Increased $\mathrm{WBC} \uparrow$ count & $9(64.3 \%)$ \\
\hline & Increased CRP\$ level & $9(64.3 \%)$ \\
\hline & Decreased $\mathrm{SpO}_{2} \S$ level & $7(50.0 \%)$ \\
\hline Radiological findings & Exacerbations & $10(71.4 \%)$ \\
\hline
\end{tabular}

$\dagger \mathrm{WBC}=$ white blood cells, Increased WBC count was defined as over 9700/ $\mu$ l. $\ddagger$ CRP $=$ C-reactive protein, $\S \mathrm{SpO}_{2}=$ oxygen saturation measured using an oximeter.

which was lower than that reported in a previous survey (1990).

HP is rare disease for a community hospital. According to a previous report, [15] the overall age-adjusted death rates of HP increased significantly between 1980 and 2002, from 0.09 to 0.29 per million. Thus, since our hospital covered approximately 4 million people in Tokyo, the annual number of patients is estimated less than one. We collected data on almost all cases in our hospital in the last 10 years. Although many cases of HP have been reported previously, the reported cases included cases from multicenter studies, such as a survey performed in Japan, [4] or from a report of a large outbreak at company. [16] The strength of this study is that all the cases were diagnosed and managed at a single institute under the supervision of academicians. The main objective of this study was to investigate the problems in interaction between primary care physicians and chest specialists, and this is the first study in this regard on HP.

Prior to discussing the data, the characteristics and problems of this study design should be pointed out. An advantage of the present study was that it included many patients treated at a single institute over 10 years who underwent extensive testing, including HRCT, BALF analysis, transbronchial lung biopsy, and detection of novel biomarkers such as KL-6, to establish a diagnosis of HP. A previous survey of 835 patients from 168 different hospitals involved diagnostic criteria that did not include all of these recent examination procedures [4]. However, a disadvantage of our present study was that pulmonary function tests were performed on a limited number of patients; therefore, we did not observe a difference in the results of lung function tests in this study. Unfortunately, data for serum KL-6 levels were not available for all of our cases.

The present data clarified several novel and interesting observations. First, it should be noted that all patients were initially treated on the basis of a different diagnosis in primary practice. This was primarily because of the fact that the initial symptoms of HP are similar to those of other respiratory diseases such as common cold or bronchial asthma. In such cases, most primary physicians might hesitate to perform more complicated and expensive examinations such as HRCT, KL-6 detection, or detection of serum precipitins. However, these data combined with the results of BALF analysis and HRCT might be useful for diagnosis. These findings suggest that a close collaboration between general practitioners and staff at specialized hospitals is essential for early diagnosis and adequate treatment. We also recommend that instead of complicated criteria [6], a simple manual or collection of clinical guidelines be created for non-specialists in Japan, although several different diagnostic criteria for HP have been proposed $[1,7,17]$. Problems arising in the diagnoses of various types of HP, such as subclinical or chronic illnesses, should be emphasized; however, the level of detail currently available for such information is still insufficient (see below) [9]. A previous study also showed that chest radiographs were less likely to be abnormal when a population-based approach was undertaken for the diagnosis of HP [4].

Second, the prevalence of S-HP in the present study was $64.3 \%$, which was lesser than that in a previous survey $(70 \%)$ (1990). Overall, our study findings suggest that several heterogeneous clinical phenotypes exist in S-HP cases diagnosed according to the Japanese criteria. They also indicate that cases of S-HP still include those with undetermined precipitins, which might be classified as excluded cases in this study. This could be because of the problems in the purification of precipitins, or it may suggest the existence of unknown types of HP. In this regard, further studies are required for the correct identification of other cases, which should be based on data collected using recent technology on a nationwide basis, as was done previously $[4,5]$.

Third, we attempted to determine definite cases of chronic or subacute HP by using the tools at hand. Approximately $5 \%$ of HP patients develop the chronic form of the disease [18]. An epidemiologic survey for determining chronic HP cases among 36 Japanese cases [9] revealed that the proportion of patients with S-HP who developed chronic HP was smaller than that of the pa- 
tients with bird fancier's lung. Ohtani et al. [19-21] and Inase et al. [22] reported problems in the diagnoses of patients with chronic S-HP. They further cautioned that some cases with bird fancier's lung were misdiagnosed as idiopathic interstitial pneumonia (IIP) or chronic and insidious HP before referral to their institution [19-22]. The data shown in Figure 1 indicates that the patient group comprised 3 subgroups categorized in terms of the time elapsed from the onset of initial symptoms to the time of referral to our hospital. We suspect that the cases showing a longer interval were cases of chronic illness, as has been previously reported [9]. However, no repeat episodes in the same patient were included in the present study. Imaging findings and histopathological data in chronic bird fancier's lung are reported to be similar to those in chronic S-HP [20-22]. Previous reports also show that chronic HP and interstitial pulmonary fibrosis (IPF) share several clinical characteristics, including histological findings as well as the acute exacerbation associated with diffuse lung damage [9]. Taken together, these reports suggest that the diagnosis of IPF requires exclusion of other causes of interstitial lung diseases, including environmental exposure [23]. These variations probably reflect differences in genetically controlled immunologic responses among different individuals, as well as the frequency and concentration of antigen exposure $[8,9]$. These variables remain speculative and clearly require further study.

In our study, neither data on the serum concentration of KL-6 nor radiological changes on HRCT images led to a conclusive diagnosis of HP. Serum KL-6 concentration $>1000 \mathrm{U} / \mathrm{ml}$ was highly suggestive of and compatible with a diagnosis of HP (Figure 2). The serum concentration of KL-6 is high among patients with various types of interstitial pneumonitis [24] although its sensitivity is well guaranteed. Further, ground-glass opacity or a thick interacinar septum on HRCT images was supportive of a diagnosis of HP. These observations were based on recent advances in technology using HRCT and are not described in the current guidelines [4-6]. However, the samples collected in this study were limited, and this issue clearly requires further study. The utility of inhalation provocation tests for the diagnosis of HP has been reported. These tests can be particularly helpful in distinguishing chronic HP associated with avian antigens from other diseases in differential diagnosis $[25,26]$. While these methods can usually identify individuals with HP [8], they are time-consuming, expensive, and may be difficult to interpret. They should only be conducted in specialized centers with experience in inhalation provocation techniques [8]. One current report indicates that inhalation provocation tests are not recom- mended for the routine evaluation of patients with suspected HP [7]. The return-home challenge test differs from the inhalation provocation tests for diagnosis of HP and may preserve safety; however, it raises an interpretation problem, particularly in cases of chronic HP.

In conclusion, all patients initially received treatment on the basis of a different diagnosis at a primary practice center. Interaction between primary care physicians and chest specialists is essential for solving problems associated with the early diagnosis and adequate treatment of HP.

\section{REFERENCES}

[1] Y. Cormier and Y. Lacasse, "Keys to the Diagnosis of Hypersensitivity Pneumonitis: The Role of Serum Precipitins, Lung Biopsy, and High-Resolution Computed Tomography," Clinical Pulmonary Medicine, Vol. 3, No. 2, 1996, pp. 72-77.

[2] C. Rose and T. E. King Jr., "Controversies in Hypersensitivity Pneumonitis," The American Review of Respiratory Disease, Vol. 145, No. 1, 1992, pp. 1-2. doi:10.1164/ajrccm/145.1.1

[3] M. Selman, "Hypersensitivity Pneumonitis," In: T. E. King Jr. and M. I. Schwarz, Eds., Interstitial Lung Disease, 4th Editoin, BC Decker, Hamilton, 2003, p. 452.

[4] M. Ando, K. Arima, R. Yoneda and M. Tamura, "Japanese Summer-Type Hypersensitivity Pneumonitis. Geographic Distribution, Home Environment, and Clinical Characteristics of 621 Cases," The American Review of Respiratory Disease, Vol. 144, No. 4, 1991, pp. 765-769. doi:10.1164/ajrccm/144.4.765

[5] M. Ando, K. Konishi, R. Yoneda and M. Tamura, "Difference in the Phenotypes of Bronchoalveolar Lavage Lymphocytes in Patients with Summer-Type Hypersensitivity Pneumonitis, Farmer's Lung, Ventilation Pneumonitis, and Bird Fancier's Lung: Report of a Nationwide Epidemiologic Study in Japan," Journal of Allergy and Clinical Immunology, Vol. 87, No. 5, 1991, pp. 1002 1009. doi:10.1016/0091-6749(91)90423-L

[6] K. Yoshida, M. Suga, Y. Nishiura, K. Arima, R. Yoneda, M. Tamura and M. Ando, "Occupational Hypersensitivity Pneumonitis in Japan: Data on a Nationwide Epidemiological Study," Occupational andEnvironmental Medicine, Vol. 52, No. 9, 1995, pp. 570-574. doi:10.1136/oem.52.9.570

[7] H. B. Richerson, I. L. Bernstein, J. N. Fink, G. W. Hunninghake, H. S. Novey, C. E. Reed, J. E. Salvaggio, M. R. Schuyler, H. J. Schwartz and D. J. Stechschulte, "Guidelines for the Clinical Evaluation of Hypersensitivity Pneumonitis. Report of the Subcommittee on Hypersensitivity Pneumonitis," Journal of Allergy and Clinical Immunology, Vol. 84, No. 5, 1989, pp. 839-844. doi:10.1016/0091-6749(89)90349-7

[8] L. C. Mohr, "Hypersensitivity Pneumonitis," Current Opinion in Pulmonary Medicine, Vol. 10, No. 5, 2004, pp. 
401-411. doi:10.1097/01.mcp.0000135675.95674.29

[9] Y. Yoshizawa, Y. Ohtani, H. Hayakawa, A. Sato, M. Suga and M. Ando, "Chronic Hypersensitivity Pneumonitis in Japan: A Nationwide Epidemiologic Survey," Journal of Allergy and Clinical Immunology, Vol. 103, No. 2, 1999, pp. 315-320. doi:10.1016/S0091-6749(99)70507-5

[10] N. Kohno, "Serum Marker KL-6/MUC1 for the Diagnosis and Management of Interstitial Pneumonitis," Journal of Investigative Medicine, Vol. 46, No. 3-4, 1999, pp. 151158.

[11] L. S. Newman, K. Kreiss, T. E. King Jr., S. Seay and P. A. Campbell, "Pathologic and Immunologic Alterations in Early Stages of Beryllium Disease: Reexamination of Disease Definition and Natural History," American Review of Respiratory Disease, Vol. 139, No. 6, 1989, pp. 1479-1486.

[12] M. L. Muilenberg, "Aeroallergen Assessment by Microscopy and Culture," Immunology and Allergy Clinics of North America, Vol. 9, No. 2, 1989, pp. 245-268.

[13] Y. Lacasse, M. Selman, U. Costabel, J. C. Dalphin, M. Ando, F. Morell, R. Erkinjuntti-Pekkanen, N. Muller, T. V. Colby, M. Schuyler, Y. Cormier and HP Study Group, "Clinical Diagnosis of Hypersensitivity Pneumonitis," American Journal of Respiratory and Critical Care Medicine, Vol. 168, No. 8, 2003, pp. 952-958.

doi:10.1164/rccm.200301-1370C

[14] C. S. Glazer, C. S. Rose and D. A. Lynch, "Clinical and Radiologic Manifestations of Hypersensitivity Pneumonitis," Journal of Thoracic Imaging, Vol. 17, No. 4, 2002, pp. 261-272. doi:10.1097/00005382-200210000-00003

[15] K. M. Bang, D. N. Weissman, G. A. Pinheiro, V. C. Antao, J. M. Wood and G. Syamlal, "Twenty-Three Years of Hypersensitivity Pneumonitis Mortality Surveillance in the United States," American Journal of Industrial Medicine, Vol. 49, No. 12, 2006, pp. 997-1004. doi:10.1002/ajim.20405

[16] K. H. Dangman, S. R. Cole, M. J. Hodgson, C. Kuhn, M. L. Metersky, P. Schenck and E. Storey, "The Hypersensitivity Pneumonitis Diagnostic Index: Use of Non-invasive Testing to Diagnose Hypersensitivity Pneumonitis in Metalworkers," American Journal of Industrial Medicine, Vol. 42, No. 2, 2002, pp. 150-162. doi:10.1002/ajim.10089

[17] M. J. Hodgson, D. K. Parkinson and M. Karpf, "Chest X-Rays in Hypersensitivity Pneumonitis: A Metaanalysis of Secular Trend," American Journal of Industrial Medicine, Vol. 16, No. 1, 1989, pp. 45-53.

\section{doi:10.1002/ajim.4700160106}

[18] E. Pozzi, "Extrinsic Allergic Alveolitis (Hypersensitivity pneumonitis)," In: C. Grassi, C. Brambella, U. Costabel, R. A. Stockley, R. Naeije and R. Rodriguez-Roisin, Eds., Pulmonary Diseases, McGraw-Hill International (UK) Ltd, London, 1999, pp. 289-294.

[19] Y. Ohtani, J. Ochi, K. Mitaka, T. Takemura, T. Jinta, J. Kuramochi, Y. Miyazaki, N. Inase and Y. Yoshizawa, "Chronic Summer-Type Hypersensitivity Pneumonitis Initially Misdiagnosed as Idiopathic Interstitial Pneumonia," Internal Medicine, Vol. 47, No. 8, 2008, pp. 857 862. doi:10.2169/internalmedicine.47.0656

[20] Y. Ohtani, S. Saiki, Y. Sumi, N. Inase, S. Miyake, U. Costabel and Y. Yoshizawa, "Clinical Features of Recurrent and Insidious Chronic Bird Fancier'S Lung," Annals of Allergy, Asthma \& Immunology, Vol. 90, No. 6, 2003, pp. 604-610. doi:10.1016/S1081-1206(10)61863-7

[21] Y. Ohtani, S. Saiki, M. Kitaichi, Y. Usui, N. Inase, U. Costabel and Y. Yoshizawa, "Chronic Bird Fancier'S Lung: Histopathological and Clinical Correlation. An Application of the 2002 ATS/ERS Consensus Classification of the Idiopathic Interstitial Pneumonias," Thorax, Vol. 60, No. 8, 2005, pp. 665-671. doi:10.1136/thx.2004.027326

[22] N. Inase, Y. Ohtani, Y. Usui, Y. Miyazaki, T. Takemura and Y. Yoshikawa, "Chronic Summer-Type Hypersensitivity Pneumonitis: Clinical Similarities to Idiopathic Pulmonary Fibrosis," Sarcoidosis Vasculitis Diffuse Lung Diseases, Vol. 24, No. 2, 2007, pp. 141-147.

[23] K. Arima, M. Ando, K. Ito, T. Sakata, T. Yamaguchi, S. Araki, M. Futatsuka, "Effect of Cigarette Smoking on Prevalence of Summer-Type Hypersensitivity Pneumonitis Caused by Trichosporon Cutaneum," Archives of Occupational and Environmental Health, Vol. 47, No. 4, 1992, pp. 274-278. doi:10.1080/00039896.1992.9938361

[24] B. van den Blink, M. S. Wijsenbeek and H. C. Hoogsteden, "Serum Biomarkers in Idiopathic Pulmonary Fibrosis," Pulmonary Pharmacology and Therapeutics, Vol. 23, No. 6, 2010, pp. 515-520. doi:10.1016/j.pupt.2010.08.001

[25] A. Ramírez-Venegas, R. H. Sansores, R. Pérez-Padilla, G. Carrillo and M. Selman, "Utility of a Provocation Test for Diagnosis of Chronic Pigeon Breeder's Disease," American Journal of Respiratory and Critical Care Medicine, Vol. 158, No. 3, 1998, pp. 862-869.

[26] Y. Ohtani, K. Kojima, Y. Sumi, M. Sawada, N. Inase, S. Miyake and Y. Yoshizawa, "Inhalation Provocation Tests in Chronic Bird Fancier's Lung," Chest, Vol. 118, No. 5, 2000, pp. 1382-1389. doi:10.1378/chest.118.5.1382 